\title{
Acidose Tubular Renal Distal: Expressão Clínica Variável na Mesma Família
}

\author{
Distal Renal Tubular Acidosis: Clinical Variability in \\ the Same Family
}

\author{
Daniela RAMOS $\square^{1}$, Sofia REIS ${ }^{2}$, Carolina CORDINHÃ ${ }^{3}$, Carmen do CARMO ${ }^{3}$, Clara GOMES ${ }^{3}$, \\ António Jorge CORREIA ${ }^{3}$ \\ Acta Med Port 2019 Jul-Aug;32(7-8):542-545 • https://doi.org/10.20344/amp.10758
}

\section{RESUMO}

A acidose tubular renal distal primária deve-se a um defeito genético caracterizado pela incapacidade de acidificar a urina. A sintomatologia é inespecífica e muito variável. Descrevem-se seis casos de acidose tubular renal distal numa família em que a doença afetou quatro gerações. O primeiro caso foi diagnosticado aos três anos por hematúria e urolitíase. Posteriormente foram estudados a irmã, os dois filhos e dois sobrinhos do caso índex. Apesar de assintomáticos, todos apresentavam nefrocalcinose e acidose metabólica hiperclorémica, à exceção de um caso com gasimetria normal mas com nefrocalcinose e incapacidade de acidificação urinária. Na evolução todos mantiveram nefrocalcinose, o caso índex desenvolveu hipertensão arterial mas nenhum evoluiu para insuficiência renal crónica. O diagnóstico da acidose tubular renal distal autossómica dominante é geralmente mais tardio e com sintomatologia mais ligeira. A doença pode contudo manifestar-se precocemente e com espectro de gravidade variável. O rastreio pela história familiar permite antecipar o diagnóstico e iniciar tratamento mais precocemente.

Palavras-chave: Acidose Tubular Renal Distal/genética; Criança

\section{ABSTRACT}

Primary distal renal tubular acidosis is a genetic disorder characterized by the inability in acidification of urine. Symptoms are usually non-specific and highly variable. We described six cases in a family with four generations affected. The first case was diagnosed in a 3-year-old child presenting with hematuria and urolithiasis. Later, his sister, sons and two nephews were studied. Although asymptomatic, they all had nephrocalcinosis and hyperchloremic metabolic acidosis with normal anionic gap, except one case with normal arterial blood gas test but with nephrocalcinosis and inability of urinary acidification. At follow-up, they all maintained nephrocalcinosis, the index case had acute renal damage and developed hypertension, but none developed chronic renal disease. The diagnosis of autosomal dominant distal renal tubular acidosis is generally made later and patients tend to present with milder disease. But the condition may manifest early and have a variable phenotypic severity spectrum. Carrying out screening through assessment of family history enables an earlier diagnosis while also allowing treatment to start sooner.

Keywords: Acidosis, Renal Tubular/genetics; Child

\section{INTRODUÇÃO}

A acidose tubular renal distal (ATRd) é uma condição crónica que se caracteriza por acidose metabólica hiperclorémica com hiato aniónico normal, hipocaliémia e hipercalciúria. Deve-se à incapacidade das células do túbulo contornado distal e coletor promoverem a excreção urinária de $\mathrm{H}^{+}$. O pH urinário mantém-se elevado $(>5,5)$ apesar da acidose metabólica. ${ }^{1-5}$

A acidose crónica conduz a aumento da reabsorção óssea. $O$ excesso de cálcio que é libertado no sangue associado à diminuição da expressão tubular renal de transportadores de cálcio, na acidose metabólica, causam hipercalciúria. $^{3}$

A forma primária de ATRd é a mais comum em idade pediátrica. Deve-se a defeitos genéticos nos mecanismos de transporte (mutações no trocador de cloro-bicarbonato (AE1) ou na bomba $\mathrm{H}^{+}$-ATPase), com um padrão de transmissão autossómico recessivo ou dominante. ${ }^{1,6-10}$ As formas autossómicas dominantes (AD) cursam geralmente com acidose mais ligeira e apresentação mais tardia enquanto as formas recessivas são mais precoces e com clínica mais grave, ${ }^{2,5,11}$ podendo associar-se a surdez neurossensorial bilateral progressiva e irreversível. ${ }^{1,2,5,7,11}$

As manifestações clínicas mais frequentes são má progressão estato-ponderal, vómitos, diarreia ou obstipação, poliúria e desidratação..$^{1,3,5} \mathrm{~A}$ desmineralização óssea pode levar ao raquitismo e osteomalacia. ${ }^{3,11}$ Evolui quase sempre com nefrocalcinose e urolitíase $e^{2,5,6,8,11}$ que podem progredir para doença renal crónica. ${ }^{1,4,7,9}$

O tratamento baseia-se na administração crónica de suplementos alcalinos, sob a forma de bicarbonato ou citrato. ${ }^{1,3,11}$

Apresentam-se seis casos de ATRd, de uma mesma família, seguidos na consulta de Nefrologia Pediátrica de um hospital terciário.

\section{CASOS CLÍNICOS}

Conforme está representado no genograma (Fig. 1), a doença afetou quatro gerações de uma família oriunda do interior centro de Portugal, revelando um padrão de hereditariedade $A D$, com risco de recorrência de $50 \%$ para os descendentes. Não havia história de consanguinidade.

1. Serviço de Pediatria Médica. Hospital Pediátrico de Coimbra. Centro Hospitalar e Universitário de Coimbra. Coimbra. Portugal.

2. Serviço de Pediatria. Centro Hospitalar Tondela-Viseu. Viseu. Portugal.

3. Unidade de Nefrologia Pediátrica. Hospital Pediátrico de Coimbra. Centro Hospitalar e Universitário de Coimbra. Coimbra. Portugal.

$\square$ Autor correspondente: Daniela Ramos. danielaframos88@gmail.com

Recebido: 05 de maio de 2018 - Aceite: 21 de agosto de 2018 | Copyright $\odot$ Ordem dos Médicos 2019 


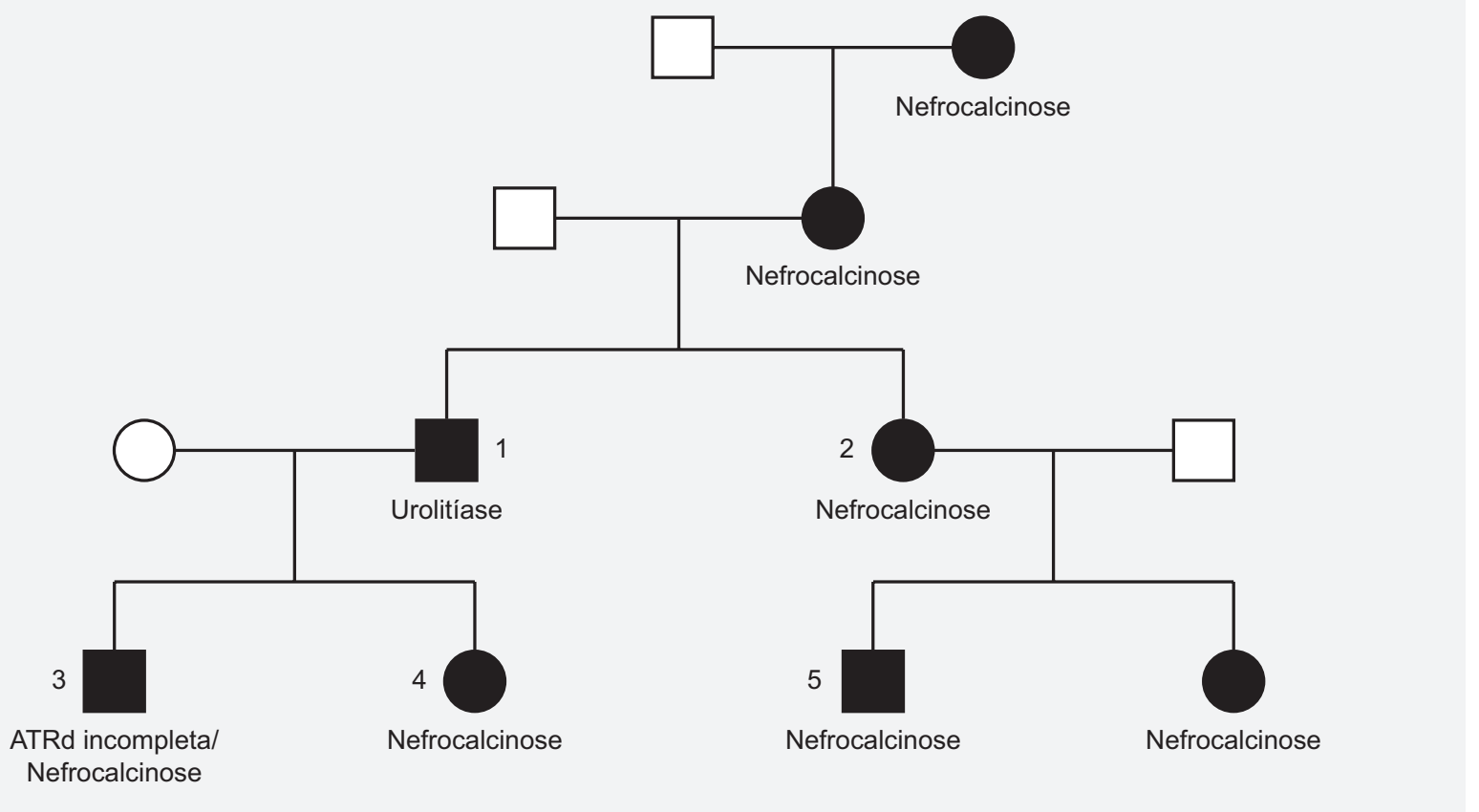

Figura 1 - Genograma da família com acidose tubular renal distal

Descrevem-se os seis casos assinalados no genograma, seguidos na nossa consulta, cujas manifestações clínicas e evolução se resumem na Tabela 1. Nos antecedentes familiares os casos das duas primeiras gerações tinham o diagnóstico de nefrocalcinose e evoluíram sem doença renal crónica terminal.

$O$ caso $n^{\circ} 1$ era um doente do sexo masculino, que aos três anos de idade iniciou episódios de hematúria macroscópica recorrente associada a litíase. Da investigação constatou-se acidose metabólica com hiato aniónico normal, hipercalciúria, oxalúria, cistinúria, uricosúria e citratúria normais. O teste de furosemida confirmou incapacidade de acidificação da urina. A radiografia simples do abdómen e a ecografia renal revelaram nefrocalcinose medular bilateral e litíase à esquerda. Iniciou tratamento com citrato de potássio com normalização da calciúria e da acidose. Aos dez anos iniciou episódios de cólica renal, um deles complicado de lesão renal aguda de etiologia obstrutiva, com cálculo no ureter esquerdo e outro à saída de um cálice inferior à direita. Colocou cateter ureteral esquerdo e fez sessões de litotrícia com normalização da função renal no prazo de um mês. Aos 14 anos foi diagnosticada hipertensão arterial. Teve alta nesta idade com função renal normal sob terapêutica com citrato de potássio e losartan-hidroclorotiazida. Fez estudo genético não tendo sido identificada mutação do gene $S L C 4 A 1$, a mais frequente na forma dominante de ATRd.

Pela história familiar foi investigada a irmã com oito anos (caso $n^{\circ} 2$ ), que apresentava também acidose metabólica com hiato aniónico normal e nefrocalcinose medular bilateral. Iniciou tratamento com citrato de potássio com normalização do equilíbrio ácido-base, mantendo nefrocalcinose,

Tabela 1 - Resumo das características clínicas, laboratoriais, ecográficas e evolução dos casos

\begin{tabular}{|c|c|c|c|c|c|c|c|c|c|c|c|c|}
\hline \multirow[b]{2}{*}{ Caso } & \multicolumn{8}{|c|}{ Diagnóstico } & \multicolumn{4}{|c|}{ Evolução } \\
\hline & Idade & Sintoma & $\begin{array}{c}\text { AM } \\
\text { c/ HA } \\
\text { normal }\end{array}$ & $\mathrm{pH} \mathrm{U}$ & $\begin{array}{c}\mathrm{pH} / \mathrm{HCO}_{3} \\
\text { sérico }\end{array}$ & $\begin{array}{c}\mathrm{Ca} / \mathrm{Cr} \\
\text { (U) }\end{array}$ & FR & Nefrocalcinose & Idade $^{* *}$ & Nefrocalcinose & HTA & DRC \\
\hline 1 & $3 \mathrm{~A}$ & $\begin{array}{c}\text { He- } \\
\text { matúria } \\
\text { Cálculo } \\
\text { renal }\end{array}$ & Sim & * & * & $\uparrow$ & $\mathrm{N}$ & $\begin{array}{c}\text { Sim } \\
\text { (+Litíase) }\end{array}$ & $14 \mathrm{~A}$ & $\begin{array}{c}\text { Sim } \\
\text { (+Litíase) }\end{array}$ & Sim & Não \\
\hline 2 & $8 \mathrm{~A}$ & - & Sim & * & * & $\mathrm{N}$ & $\mathrm{N}$ & Sim & $16 \mathrm{~A}$ & Sim & Não & Não \\
\hline 3 & $15 \mathrm{M}$ & - & Não & 7 & $7,34 / 23,2$ & $\mathrm{~N}$ & $\mathrm{~N}$ & Sim & $31 \mathrm{M}$ & Sim & Não & Não \\
\hline 4 & $1 \mathrm{M}$ & - & Sim & 7,75 & $7,1 / 14,3$ & $\uparrow$ & $\mathrm{N}$ & Sim & $23 \mathrm{M}$ & Sim & Não & Não \\
\hline 5 & $6 \mathrm{~A}$ & - & Sim & 7,42 & $7,31 / 22,7$ & $\uparrow$ & $\mathrm{N}$ & Sim & $7 \mathrm{~A}$ & Sim & Não & Não \\
\hline 6 & $4 \mathrm{M}$ & - & Sim & 8 & $7,3 / 16,2$ & $\mathrm{~N}$ & $\mathrm{~N}$ & Sim & $30 \mathrm{M}$ & Sim & Não & Não \\
\hline
\end{tabular}

A: anos; AM: acidose metabólica; Ca: cálcio; Cr: creatinina; DRC: doença renal crónica; FR: função renal; HÁ: hiato aniónico; HTA: hipertensão arterial; M: meses; Tx: terapêutica alcalinizante; U: urinário. Idade $^{* *}=$ idade na última avaliação; ${ }^{*}=$ resultado não disponível 
sem visualização de cálculos. Manteve-se sempre assintomática até à data da alta (16 anos), com tensão arterial e função glomerular normais.

Os filhos dos casos $n^{0} 1$ (1 e 15 meses) e do $n^{\circ} 2$ (4 meses e 6 anos) foram investigados também por rastreio familiar. Apesar de assintomáticos, com boa evolução ponderal e sem surdez, todos apresentavam acidose metabólica com hiato aniónico normal, à exceção do caso $n^{\circ} 3$. Neste confirmou-se incapacidade de acidificação da urina no teste de furosemida. Todos tinham nefrocalcinose medular bilateral (Fig. 2) incluindo a pequena lactente com um mês, apesar de apenas se ter documentado hipercalciúria nos casos $n^{\circ} 4$ e 5 . Nenhum caso apresentou hipocaliémia ou anemia.

Todos iniciaram terapêutica alcalinizante com bicarbonato de sódio ou citrato de potássio com correção da acidose e da hipercalciúria. Na última avaliação sob terapêutica alcalinizante, mantinham nefrocalcinose estável sem litíase, tensão arterial e função renal normais, com crescimento e desenvolvimento adequados à idade.

\section{DISCUSSÃO}

AATRd é uma patologia rara e o seu diagnóstico requer um alto índice de suspeição dada a grande variabilidade clínica e inespecificidade dos sintomas. A história familiar deve também ser valorizada. ${ }^{4}$ Nesta família, depois do diagnóstico do caso $n^{\circ} 1$, no contexto de hematúria e urolitíase, foi possível fazer o diagnóstico dos outros casos descritos, que apesar de assintomáticos já apresentavam alterações analíticas e ecográficas.

A forma $A D$ geralmente tem um diagnóstico mais tardio e sintomatologia mais ligeira, ${ }^{4,5,10-13}$ podendo contudo manifestar-se precocemente, com espectro de gravidade fenotípica variável. ${ }^{9,10} \mathrm{~A}$ acidose é descrita como mais ligeira em comparação com as formas recessivas, ${ }^{5}$ mas todas apresentam incapacidade de acidificação urinária e nefrocalcinose precoce. ${ }^{5} \mathrm{O}$ caso $\mathrm{n}^{\circ} 3$, sem acidose, apresentava nefrocalcinose e pela prova de furosemido confirmou-se uma ATRd incompleta com incapacidade de acidificar a urina. ${ }^{1,2,13}$ Raramente se constata doença óssea tal como nesta série. ${ }^{5}$

AATRd AD tem sido associada a mutações no transportador cloro-bicarbonato codificado pelo gene SLC4A1.11,13 Esta mutação não foi identificada no caso $\mathrm{n}^{\circ} 1$ desta família mas a sequenciação genética convencional não encontra mutações em até $20 \%-25 \%$ dos doentes. ${ }^{2}$

A acidose crónica não tratada pode levar a raquitismo, osteomalácia e litíase.4,11 Atrasos no diagnóstico têm implicações no crescimento e desenvolvimento da criança. ${ }^{9}$ Os doentes adequadamente tratados, geralmente são assintomáticos, sendo o prognóstico excelente, sobretudo quando iniciam tratamento precoce..$^{1,3,13} \mathrm{~A}$ maioria das complicações são resolvidas com o controle da acidose exceto a nefrocalcinose que geralmente não regride,,$^{1,7,9,13}$ como

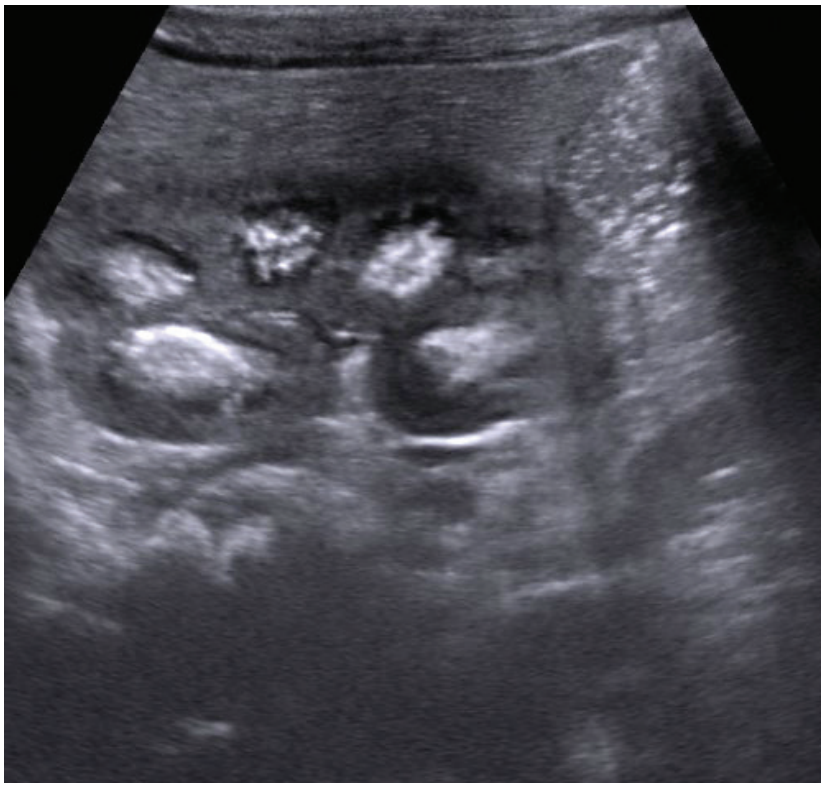

Figura 2 - Nefrocalcinose medular no caso $n^{\circ} 4$ (um mês de idade)

nos casos apresentados. A deterioração da função renal ocorre raramente e está relacionada com persistência de hipercalciúria e litíase recorrente. ${ }^{1}$

Os autores pretendem mostrar a grande variabilidade clínica desta patologia, sem correlação genótipo-fenótipo, como demonstrado nesta família. O rastreio familiar com a realização de uma gasimetria e ecografia renal são fundamentais para o diagnóstico e tratamento precoces. O estudo genético destas famílias pode estabelecer o risco de recorrência nos descendentes.

\section{PROTECÇÃO DE PESSOAS E ANIMAIS}

Os autores declaram que os procedimentos seguidos estavam de acordo com os regulamentos estabelecidos pelos responsáveis da Comissão de Investigação Clínica e Ética e de acordo com a Declaração de Helsínquia da Associação Médica Mundial.

\section{CONFIDENCIALIDADE DOS DADOS}

Os autores declaram ter seguido os protocolos do seu centro de trabalho acerca da publicação de dados.

\section{CONSENTIMENTO INFORMADO}

Obtido.

\section{CONFLITOS DE INTERESSE}

Os autores declaram não ter qualquer conflito de interesse relativamente ao presente artigo.

\section{FONTES DE FINANCIAMENTO}

Não foi utilizada nenhuma bolsa ou subsídio para a realização do trabalho. 


\section{REFERÊNCIAS}

1. Soriano JR. Renal tubular acidosis: the clinical entity. Frontiers in nephrology. J Am Soc Nephrol. 2002;13:2160-70.

2. Santos F, Ordonez FA. Clinical and laboratory approaches in the diagnosis of renal tubular acidosis. Pediatr Nephrol. 2015;30:2099-107.

3. Besouw MT, Bienias M, Walsh P, Kleta R, Van't Hoff WG, Ashton E, et al. Clinical and molecular aspects of distal renal tubular acidosis in children. Pediatr Nephrol. 2017;32:987-96.

4. Boualla L, Jdioui W, Soulami K, Ratbi I, Sefiani A. Clinical and molecular findings in three Moroccan families with distal renal tubular acidosis and deafness: Report of a novel mutation of ATP6V1B1 gene. Curr Res Transl Med. 2016;64:5-8.

5. Mattoo TK. Etiology and clinical manifestations of renal tubular acidosis in infants and children. UpToDate, last updated in Jun 02, 2017. [accessed 2018 Apr 20]. Available from: https://www.uptodate.com/ contents/etiology-and-clinical-manifestations-of-renal-tubular-acidosisin-infants-and-children.

6. Bajaj G, Quan A. Renal tubular acidosis and deafness: report of a large family. Am J Kidney Dis. 1996;27:880-2.

7. Naeem A, Kiblawi MA, Kar SS, Ahmed E, Mossad S, Goud BK. Familial renal tubular acidosis: report of two cases from a single family. Ann
Nigerian Med. 2015;9:33-7.

8. Khositseth S, Sirikanerat A, Wongbenjarat K, Opastirakul S, Khoprasert $S$, Peuksungnern $R$, et al. Distal renal tubular acidosis associated with anion exchanger 1 in children in Thailand. Am J Kidney Dis. 2007;49:841-50.

9. Periquito I, Anaxore C, Santo CE, D'Elia C, Abranches M, Castro I. Acidose tubular renal distal e surdez neurossensorial com mutação no gene ATP6V1B1. Port J Nephrol Hypert. 2013;27:109-12.

10. Ito N, Ihara K, Kamoda T, Akamine S, Kamezaki K, Tsuru N, et al. Autossomal dominant distal renal tubular acidosis caused by a mutation in the anion exchanger 1 gene in a Japanese family. CEN Case Report. 2015;4:218-22.

11. Karet F. Inherited distal renal tubular acidosis. J Am Soc Nephrol. 2002;13:2178-84.

12. Palazzo V, Provenzano A, Becherucci F, Sansavini G, Mazzinghi B, Orlandini $\mathrm{V}$, et al. The genetic and clinical spectrum of a large cohort of patients with distal renal tubular acidosis. Kidney Int. 2017; 91:1243-55.

13. Silva AC, Lima CJ, Souto MF. Acidose tubular renal em pediatria. J Bras Nefrol. 2007;29:38-47. 